\title{
Consideraciones en redacción científica: la introducción y los métodos
}

\section{Scientific writing considerations: introduction and methods}

\section{Resumen}

Redactar artículos científicos implica conocer reglas y protocolos relacionados a la argumentación, explicación, ortografía, estilo y edición de textos. Escribir y leer apropiadamente artículos científicos son habilidades clave para los investigadores. Clásicamente los artículos originales presentan como estructura general: introducción, métodos, resultados y discusión (IMRyD). Todos los segmentos presentan características propias y normas de redacción que son valoradas por los editores y revisores; valoraciones que decidirán la aceptación o rechazo de un manuscrito para ser publicado en una revista científica. En la presente nota científica se resumen algunas recomendaciones para redactar apropiadamente una introducción y los métodos de un artículo científico.

Palabras clave: Comunicación y divulgación científica; Escritura médica; Métodos; Odontología; Publicaciones de divulgación científica.

\begin{abstract}
Scientific writing involves the knowledge of the rules and protocols related with argumentation, explanation, spelling, style and text editing. Writing and reading scientific papers are key skills for researchers. Classically, original articles present as a general structure: introduction, methods, results and discussion (IMRaD). All topics present their own characteristics and writing standards that are assessed by the editors and reviewers; these assessments will decide the acceptance or rejection of a manuscript for publication in a scientific journal. This scientific note summarizes some suggestions for properly writing an introduction and the methods of a scientific article.
\end{abstract}

Keywords: Dentistry; Medical writing; Publications for science diffusion; Methods; Scientific communication and diffusion.

\section{Nota Científica}

Yuri Castro-Rodríguez 1,2,a , Manuel Antonio Mattos-Vela ${ }^{2, b}$, Aron Aliaga-Del Castillo ${ }^{3, c}$

${ }^{1}$ Universidad Privada Juan Pablo II.

2 Universidad Nacional Mayor de San Marcos. Lima, Perú.

${ }^{3}$ Universidad de São Paulo. Facultad de Odontología de Bauru. São Paulo, Brasil.

a Maestro en Educación

${ }^{\mathrm{b}}$ Doctor en Estomatología

c Maestro en Ortodoncia

Correspondencia:

Yuri Castro-Rodríguez

Correo electrónico: yuricastro_16@hotmail.com Jr. Tomás Catari 463, Urb. El Trébol. Dpto. 201. Lima 39, Perú.

Conflicto de intereses: Los autores declaran no tener conflictos de interés.

Fuente de financiamiento: Autofinanciado

Fecha de recepción: 15/04/18

Fecha de aceptación: 01/06/18 


\section{Introducción}

La investigación es el acto de resolver y encontrar la solución a un problema; en el caso de la ciencia el enfoque está dirigido a la investigación científica.

La obtención de un resultado o una respuesta a la hipótesis planteada no culmina una investigación sino que el proceso continúa hasta alcanzar la difusión de los resultados hacia una comunidad científica. La comunicación de una investigación científica se realiza a través del proceso de producción científica; esto implica el conocimiento y aplicación de técnicas y métodos de argumentación y redacción científica así como de los géneros discursivos que permitan plasmar en el papel los hallazgos de una investigación.

Los principales medios de comunicación son los artículos científicos publicados en revistas indizadas. Dichas revistas exigen protocolos de redacción que los autores deben conocer para poder adaptar sus manuscritos para envío, y tal vez lograr la aceptación de su artículo. Tradicionalmente un artículo científico presenta como estructura: la introducción, métodos, resultados y discusión (IMRyD).

La introducción de un artículo científico permite a los autores contextualizar el tema de estudio, presentar los antecedentes, fundamentar la importancia y declarar el objetivo del estudio. Los métodos hacen mención a los procedimientos, técnicas, materiales e instrumentos que se utilizaron para encontrar la solución al problema. Ambos segmentos presentan características particulares y distintos protocolos de redacción científica, es por ese motivo que en la presente nota científica se resumen las principales consideraciones en la redacción de los segmentos "Introducción" y "Métodos" que componen un artículo científico.

\section{La introducción}

Este segmento describe el tema central (problema a investigar), la importancia del estudio, los principales antecedentes, y formula el objetivo de la investigación ${ }^{1}$. Por lo general es la "carta de presentación” del artículo hacia el lector y permite obtener una idea precisa del contenido del mismo ${ }^{2}$. Debe suministrar los suficientes antecedentes para que el lector comprenda los resultados sin necesidad de ubicar otras publicaciones ${ }^{3}$ (Tabla 1 y Figura 1). En este segmento los autores deben comunicar por qué el tema a tratar es importante y merece ser estudiado.

La redacción de sus párrafos debe responder a las preguntas: ¿Cuál es el problema?, ¿Por qué se realizó la investigación?, ¿Cuál es el objetivo de la investigación? ${ }^{7}$. Al mismo tiempo el contenido debe motivar al lector la comprensión de la investigación ${ }^{4}$. El lector debe advertir que frente a él se encuentra una información original o novedosa ${ }^{4}$. Si bien enuncia antecedentes relacionados al problema, no deben ser exagerados pues asemejará a un marco teórico; solo citar directamente las referencias pertinentes ${ }^{6}$ y considerar que en la discusión se podrá ampliar los alcances de resultados anteriores o similares ${ }^{4}$.

De preferencia los antecedentes deben ser actuales ${ }^{2}$, aunque también es plausible el uso de referencias clásicas y/o vigentes. Los autores deben valorar las referencias que consideren más importantes al momento de redactar este segmento ${ }^{3}$.

Por lo general el último párrafo de esta sección debe exponer el objetivo general y la hipótesis tentativa del estudio ${ }^{7}$. Es posible plantear varios objetivos en tal caso deben identificarse a través de números arábigos 8 (Tabla 2).

La mayoría de párrafos se redactarán en presente pues se referirán al problema a plantear ${ }^{3}$. En caso se citen a otros autores, estas oraciones deberán redactarse en pasado; se recomienda que las citaciones se realicen con superíndices antes del punto que culmine el párrafo u oración citada, aunque esta consideración dependerá del estilo de cada revista. Para realizar la citación de autores se utilizará el superíndice luego del apellido; ejemplo: Torres $\stackrel{8}{ }$ menciona...; cuando se citen dos autores se mencionarán los apellidos de ambos; ejemplo: Torres y Gómez ${ }^{8}$ mencionan...; en casos de tres o más autores se anotará el apellido del primer autor seguido del término "et al."; por ejemplo: Torres et al. ${ }^{8}$ mencionan...

Tabla 1. Consideraciones a resaltar al momento de redactar la introducción

\begin{tabular}{|c|c|c|}
\hline Características & Recomendaciones para su redacción & Modelos de estructura \\
\hline $\begin{array}{l}\text { - Centrarse en el tema principal y la formu- } \\
\text { lación del problema }{ }^{4} \text {. } \\
\text { - Utilizar términos comprensibles, claros y } \\
\text { concisos. } \\
\text { - Se redacta en presente, excepto las refe- } \\
\text { rencias o antecedentes que se redactan en } \\
\text { pasado }{ }^{1} \text {. } \\
\text { - El objetivo se redacta con un verbo en } \\
\text { infinitivo. } \\
\text { - Redactar los párrafos de forma secuencial } \\
\text { y que permita al lector la comprensión del } \\
\text { porqué se realizó el estudio }{ }^{4} \text {. } \\
\text { - Insertar citas bibliográficas, preferente- } \\
\text { mente escritas en superíndices y en orden } \\
\text { secuencial }{ }^{1} \text {, dependiendo del estilo de } \\
\text { cada revista. }\end{array}$ & $\begin{array}{l}\text { - Demostrar que la cuestión a tratar es } \\
\text { importante y que la hipótesis es novedosa } \\
\text { - Algunos sugieren, terminar de redactarla } \\
\text { al finalizar el artículo, }{ }^{5} \text {. } \\
\text { - Verificar exhaustivamente que no es el } \\
\text { primer estudio realizado sobre el tema } \\
\text { pues es desagradable los términos como } \\
\text { "es el primer ensayo realizado..." }{ }^{1} \text {. } \\
\text { - Justificar cada afirmación con una citación. } \\
\text { Mostrar que los conocimientos actuales no } \\
\text { permiten resolver el problema }{ }^{5} \text {. } \\
\text { - Se recomienda el uso de } 10-15 \text { referencias, } \\
\text { como mínimo }{ }^{1} \text {. } \\
\text { - Se recomienda un máximo de } 750 \text { pala- } \\
\text { bras redactados en } 3-5 \text { párrafos }{ }^{5} \text {. } \\
\text { - Evitar anticipar las conclusiones del } \\
\text { propio estudio }{ }^{6} \text {. }\end{array}$ & $\begin{array}{l}\text { - Marco teórico, planteamiento del proble- } \\
\text { ma, antecedentes y objetivo general }{ }^{2} \text {. } \\
\text { Problema de identificación y su delimi- } \\
\text { tación, hasta dónde se ha estudiado y } \\
\text { qué se necesita para aclarar lo faltante, } \\
\text { objetivos e hipótesis tentativa }{ }^{1} \text {. } \\
\text { - Antecedentes: ¿Qué se sabe del proble- } \\
\text { ma? Problema no resuelto. Porqué del } \\
\text { estudio y su justificación. Hipótesis y } \\
\text { objetivo }{ }^{7} \text {. } \\
\text { - Naturaleza y alcance del problema. } \\
\text { Antecedentes pertinentes que orienten al } \\
\text { lector. Por qué se eligió el tema y por qué } \\
\text { es importante }^{3} \text {. }\end{array}$ \\
\hline
\end{tabular}




\section{Introducción}

Primer Verbo en tiempo presente

párrafo [La producción cientifica estudiantil aborda gran cantidad de textos académicos; principalmente monografias, tesis, artículos científicos, grupos de estudio y creación de revistas científicas menciona

el tema central

estudiantiles. $\uparrow$ Espacios entre párrafos que otorguen $\downarrow$ cohesión textual

Citaciones que soporten las

afirmaciones

Respecto a las tesis, en la mayoria de universidades estas son/el primer acercamiento que tiene un estudiante hacia la investigación cientifica. Muchas vedes esta permite acceder a un Mención título profesional e iniciarse en el campo de la investigación ${ }^{1}$. Son muchos los estudios que del
problema elaboran perfiles bibliométricos de las tesis universitarias, y pocos estudios se han dedicado a problema investigar analizar la cantidad de tesis que finalmente llegan a comunicarse en canales formales como las revistas científicas. De estos estudios podemos citar a Osada et al. ${ }^{2}$ quienes en una evaluación de 93 tesis sustentadas entre 1998-2008, concluyen que sół el $11 \%$ fueron publicadas. Valle y Salvador ${ }^{3}$, también en 93 tesis encuentran que la principal femática de investigación son las áreas clínica $(60 \%)$ y quirúrigiea- $(19 \%)$ y sólo el $11 \%$ de tesls fue publicada en una revista indizada (1/10 tesis).

Antecedentes que avalen el problema a investigar

[Se manifiesta un problema en el proceso de comunicación científica. La producción científica culmina con el proceso de comunicación o publicación cientifica; siendo los principales canales

Mención del por de publicación las revistas científicas a través de artículos científicos ${ }^{4,5}$. Si los resultados de las investigaciones no se publican o no se divulgan para conocimiento, análisis, validación o refutación en el ámbito de la comunidad científica, es como si no se hubieran realizado. Los esfuerzos y los escasos recursos resultan siendo malgastados; agregar a esto que la gran mayoria de docentes no están preparados para afrontar tareas de investigación científica ${ }^{6-8}$ icitaciones consecutivas

¿Qué [No publicar una investigación rompe el enlace clave entre el investigador y su comunidad el problema persiste? cientifica, donde los profesionales no podrán aplicarlos y contribuir en las diferentes disciplinas de las ciencias de la salud ${ }^{9,10}$. En la presente investigación se determinó la cantidad de tesis que han sido comunicadas ên revistas cientificas por parte de los estudiantes que finalizaron la carrera de Odontologia des de los años 2005 hasta el año 2015 en la Universidad Nacional Mayor de San Marcos. Lima. Perú. Se hipotetizó que esta cantidad es baja pero quejposee una tendencia a aumentar conforme avanza la línea temporal de evaluación.

$$
\text { Citaciones }
$$$$
\text { finales }
$$

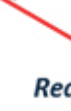

Redacción del objetivo en tiempo pasado

Redacción de la hipótesis tentativa.

Figura 1. Modelo de Introducción de un estudio titulado "Producción científica de tesis sustentadas y publicadas por estudiantes de pregrado" para una revista específica: "Revista Educación Médica".

Tabla 2. Principales características de la introducción de los artículos científicos

\begin{tabular}{|c|c|c|}
\hline Artículo original & Reporte de caso & Artículo de revisión \\
\hline $\begin{array}{l}\text { Debe presentar información científica y su } \\
\text { uso racional para el problema así como los } \\
\text { objetivos y la hipótesis. Los párrafos deben } \\
\text { responder las siguientes preguntas: ¿Cuál es } \\
\text { el problema tratado en la investigación? } \\
\text { ¿Cuáles son los principales referentes teóri- } \\
\text { cos del problema de investigación? ¿Cuáles } \\
\text { son algunos indicadores de que el problema } \\
\text { existe? ¿Por qué es relevante el problema? } \\
\text { ¿Cuáles son los objetivos centrales en la } \\
\text { investigación? }{ }^{9,10} \text {. }\end{array}$ & $\begin{array}{l}\text { Los párrafos deben resaltar lo inusual del } \\
\text { hallazgo, la patología, la técnica o biomate- } \\
\text { rial utilizado. Los antecedentes deben refe- } \\
\text { rirse a casos similares reportados por otros } \\
\text { autores }{ }^{1} \text {. Justificar por qué es necesario } \\
\text { reportar un caso clínico basado en una revi- } \\
\text { sión exhaustiva y una redacción sucinta }{ }^{1} \text {. }\end{array}$ & $\begin{array}{l}\text { Para revisiones de tema (revisiones no } \\
\text { sistemáticas) la introducción debe ser corta y } \\
\text { basarse en la importancia del tema escogido } \\
\text { a resumir. } \\
\text { Debe incluir el fundamento de la revisión } \\
\text { respecto a lo que se conoce del tema. Plan- } \\
\text { tear los objetivos acorde al protocolo PICOS } \\
\text { (participantes, intervenciones, comparacio- } \\
\text { nes, resultados y diseño de los estudios)*. }\end{array}$ \\
\hline
\end{tabular}

*Esta recomendación se encuentra orientada a las revisiones sistemáticas ${ }^{11}$. 


\section{Los métodos}

Este segmento se enfoca en describir las etapas del diseño metodológico del estudio ${ }^{1}$. Se redacta en tiempo pasado y debe presentar con detalle las herramientas, instrumentos, materiales, muestras, participantes, métodos, y procedimientos utilizados para la obtención de los resultados ${ }^{1}$.

Debe responder a la pregunta: ¿Cómo se ha realizado el estudio? ${ }^{4}$ explicando con detalle cómo se lograron los resultados de una forma clara de tal forma que otros investigadores puedan replicar el estudio ${ }^{12}$. El método científico exige que para que un resultado tenga valor científico debe ser reproducible ${ }^{3}$ (Tabla 3 ).

Debe existir la posibilidad de producir resultados iguales o semejantes. Es común que los revisores de manuscritos enviados a las revistas científicas se enfoquen en este segmento; las dudas en relación a la reproducibilidad de los métodos utilizados en la investigación, suelen ser causa de rechazo de manuscritos ${ }^{3}$.

Tabla 3. Consideraciones a resaltar al momento de redactar los métodos

Consideraciones
Se redacta en tiempo pretérito.
Especificar los datos sociodemográficos de la muestra estudiada.

Explicar los métodos para obtener y seleccionar la muestra.

Según el diseño escogido, explicar el cálculo del tamaño muestral.

Brindar las referencias de los métodos habituales, mientras que los métodos que han sido publicados pero son poco conocidos se deben referenciar y dar breves descripciones ${ }^{3}$.

Especificar, entre paréntesis, el nombre y dirección del fabricante de los materiales, instrumentos y medios utilizados ${ }^{3}$.

Describir las especificaciones técnicas de los procedimientos, cantidad exacta de reactivos utilizados, nombres genéricos, químicos, dosis aplicadas, nombres de proveedores ${ }^{3}$.

Identificar especímenes con género y especie según normas taxonómicas ${ }^{3}$.

Fórmulas, técnicas y/o procedimientos de uso común no son necesarios ser descritos sino solo mencionarlos ${ }^{3}$.

Cuando se diseñen experimentos en humanos y/o animales la mayoría de revistas exigen los permisos bioéticos de aprobación ${ }^{8}$.

Se recomienda el uso del verbo en impersonal para describir las acciones ${ }^{8}$.

Explicar por qué los métodos escogidos fueron los apropiados.

Utilizar conectores lógicos que indiquen una secuencia cronológica ${ }^{8}$.

Aunque no es obligatorio, es recomendado utilizar subtítulos para estructurar esta sección.

Debe culminar indicando los análisis estadísticos realizados. Describir el programa estadístico y versión utilizada, los métodos estadísticos descriptivos e inferenciales y el nivel de significancia empleado. Este párrafo debe permitir al lector experto con acceso a los datos originales verificar los resultados del estudio. En lo posible indicar cómo se cuantificaron los datos así como el análisis de precisión. Evitar basarse solo en la prueba de hipótesis estadísticas, como el uso de valores p, que no dan información sobre el tamaño del efecto y precisión de la estimación. Las referencias en este apartado deberían ser de antecedentes clásicos en lo posible ${ }^{6}$.
"Se diseñó un estudio transversal..."

"Se incluyeron a 92 niños entre 6-12 años de la provincia (comunidad) de Yauyos (distrito), caserío número 2 del departamento de Lima, Perú."

"Se incluyó a toda la población de estudio...", "Se utilizó un muestreo aleatorio simple...", "La muestra fue seleccionada a conveniencia..."

"El tamaño muestral fue determinado según una proporción esperada de casos del 0,8; un error estimado del 0,05; un nivel de confianza del $95 \%$ y una población de 500 pacientes..."

"Para la obtención del diagnóstico de fluorosis dental se utilizó el método planteado por Thylstrup-Fejerskov ${ }^{1}$..."

"Se utilizó una balanza digital (SecaGmbH 770 ®, Alemania)..."

"Se administró por vía oral tabletas de paracetamol $(500 \mathrm{mg})$ c/8 h por cinco días..." "Se extrajo una muestra de sangre periférica (5 $\mathrm{ml})$ de la parte anterior del antebrazo en tubos vacutainer recubiertos con EDTA (Biosciences ${ }^{\circledR}$, Francia) almacenándose en hielo y transportadas al laboratorio de Bioquímica"

"Se cultivaron cepas de Enterococcus faecalis ATCC 19433..."

"Para la amplificación de secuencias del ADN se utilizó la técnica de reacción en cadena de la polimerasa (PCR)..."

“Este estudio fue aprobado por la Junta de revisión institucional dependiente de Docencia e Investigación y el Comité de Bioética con código de registro: NCT 01301274"

"Para la selección de las revistas se analizó la base de datos SciELO..."

"Según la Academia Americana de Periodoncia se utilizó la clasificación de diagnósticos periodontales de 1999 por ser el más aceptado..."

"Primero se recolectó $5 \mathrm{ml}$ de sangre periférica, luego se procedió a centrifugar a $1200 \mathrm{rpm}$ por 5 minutos y finalmente se extrajo el plasma..."

"Muestra: 'ciento veinte pacientes adultos. Variable: Severidad de la lesión cariosa. Método: Observación clínica según ICDAS..."

"El procesamiento y análisis de los datos se realizó por medio del programa estadístico SPSS v 21 (SPSS Inc ${ }^{\circledR}$, USA). Para medir la correlación entre la estimación del peso y el peso real se aplicó la correlación de Pearson. Las comparaciones entre los porcentajes promedio de diferencia entre cada fórmula fueron realizadas según el análisis de varianzas ANOVA con una corrección post-hoc de Bonferroni. La prueba de chi cuadrado fue utilizada para comparar las proporciones de las estimaciones del peso dentro del 10\%, 15\% y $20 \%$ del peso real. Un $p$ inferior a 0,05 se consideró estadísticamente significativo". 
Respecto a su nombre existen múltiples denominaciones: "material y método", "materiales y métodos", "materiales y método", "metodología" y "diseño metodológico". Las normas de Vancouver recomiendan el uso de la denominación "métodos".

Si bien un artículo científico comienza con el título, resumen, introducción, etc. Es recomendable que los autores comiencen la redacción de su manuscrito a partir de los "métodos" 5 . Esto debido a que es el segmento más fácil de redactar y el que los autores presentan mayor dominio puesto que son ellos quienes realizaron el estudio.

Para describir los materiales se recomienda: describir las especificaciones técnicas y cantidades exactas. Mencionar la procedencia y método de preparación. Abstenerse de utilizar nombres comerciales para evitar publicidad intrínseca excepto en los que se conozca diferencias críticas entre otras marcas ${ }^{3}$.

Si dentro de los materiales se incluyen especies debe mencionarse si son: in vivo, nombre genérico, variedad, cepas, población.

Para describir los métodos se recomienda: presentarlos en orden cronológico; utilizar subtítulos para otorgar coherencia interna. Deben ser exactos, similares a una receta de cocina. El cómo y el cuándo debe ser muy claro y bien explicados para el lector. Se puede entregar a un colega y preguntarle si los métodos son comprensibles ${ }^{3}$. "El valor de una investigación se determina cuando los investigadores intentan reproducir o, más comúnmente, difunden los resultados de un estudio" ${ }^{13}$.

Existen variadas estructuras respecto a los segmentos que debe presentar la sección de métodos. De esta forma tradicionalmente se debe explicar: tipo de estudio, diseño metodológico, aprobación por algún comité de ética en investigación, objetos de estudio para el análisis (sujetos, cálculo muestral y proceso de selección), criterios de elección (métodos de reclutamiento), variables analizadas, procedimientos (procedimientos mayores, usar un orden lógico-temporal, detallar los métodos poco comunes), técnicas de recolección de datos y análisis estadístico (software y la versión utilizada, técnicas descriptivas, analíticas, métodos de variabilidad y nivel aceptado de significancia) ${ }^{1,7}$. Los reportes de caso clínico y artículos de revisión narrativos presentan una estructura diferente al de los artículos originales (Tabla 4).

Para ensayos clínicos su estructura debe presentar ${ }^{9}: 1$. Diseño del ensayo: descripción (paralelo, factorial u otro), el lugar (incluyendo zona de asignación), cambios importantes en los métodos después de iniciar el ensayo y su justificación. 2. Participantes: criterios, lugares de selección, fechas y consideraciones éticas. 3. Intervenciones: procedimientos de cada grupo con suficiente información que pueda ser replicado. 4. Resultados: especificación de las variables principales y secundarias, incluido cómo y cuándo se midieron. 5. Tamaño de la muestra: cómo se determinó. 6. Randomización: generación de secuencias (método para aleatorizar y tipo de aleatorización), mecanismo de asignación (pasos que se siguieron hasta lograr las asignaciones) e implementación (quién generó las secuencias, quién conocía a los participantes y quién asignó a los participantes a las intervenciones). 7. Enmascaramiento: quién quedó cegado luego de firmar las intervenciones y cómo se hizo. 8. Métodos estadísticos: para comparar lo grupos en cuanto a la variable respuesta principal y las secundarias; análisis adicional para subgrupos y análisis ajustados.

Para estudios observacionales debe incluir ${ }^{15}$ : diseño del estudio, contexto, participantes, variables, fuentes de datos/medidas, sesgos, tamaño de la muestra y métodos estadísticos.

Para revisiones sistemáticas (RS) debe incluir ${ }^{11}$ : Protocolo y registro: indicar si existe y dónde encontrarlo. Criterios de elegibilidad: describir las características específicas de los estudios (basado en el formato PICOS, duración de seguimiento), y características de los reportes (años abarcados, idioma, estado de publicación). Fuentes de información: bases de datos, fechas de búsqueda, idiomas incluidos en la búsqueda. Búsqueda: cómo se hizo la estrategia de búsqueda en por lo menos una base de datos para que pueda ser replicable. Selección de estudios: cómo se hizo (elegibilidad, inclusión en la RS e inclusión en el metanálisis, si fuese el caso). Proceso de recopilación de datos: cómo se extrajeron los datos (formularios piloto, por duplicado, de forma independiente) y cómo se confirmaron los datos. Ítems de los datos: definir las variables para las que se buscaron los datos. Riesgo de sesgo en los estudios: qué métodos se utilizaron para evaluar el riesgo de sesgo ya sea a nivel de los estudios o a nivel de los resultados. Si existiese la posibilidad de realizar un meta-análisis, se debe reportar: Medidas de resumen, especificarlas; por ejemplo: razón de riesgos, diferencia de las medias. Síntesis de resultados, cómo se combinaron los datos, incluir medidas de consistencia $\left(\mathrm{I}^{2}\right)$ para cada meta-análisis. Riesgo de sesgo a través de los estudios: cuando sea posible, analizar el sesgo de publicación o reporte selectivo. Análisis adicionales; describir los criterios utilizados para realizar análisis de subgrupos, análisis de sensibilidad, etc.

Tabla 4. Consideraciones a resaltar al momento de redactar los métodos

\section{Reporte de caso}

Sus "métodos" se denominan "Descripción del caso"

Se enfoca en describir la historia clínica con los datos relevantes del paciente ${ }^{4}$.

Su estructura incluye: Información del paciente, signos y síntomas clínicos, exámenes y diagnóstico otorgado, objetivos del tratamiento alternativas de tratamiento, intervención terapéutica, seguimiento y resultados ${ }^{14}$.

\section{Artículo de revisión}

Sus "métodos" se denominan "Revisión de la literatura".

Describe una secuencia lógica y secuencial de los temas y subtemas similar a un libro de texto ${ }^{4}$.

Carece de una estructura fija puesto que los autores son libres de indicar los títulos y subtítulos a utilizar según el tema de revisión. 


\section{Conclusiones}

La introducción de un artículo científico permite a los lectores comprender la importancia del problema de investigación y el porqué de la necesidad de encontrar sus resultados; los métodos facilitan la comprensión de las etapas realizadas en el estudio para encontrar los datos requeridos. Considerar que una introducción apropiada animará a que el lector continúe con los demás segmentos; la redacción de métodos claros y precisos facilitará que otros investigadores reproduzcan las técnicas y procedimientos que los autores han reportado.

\section{Referencias bibliográficas}

1. Sánchez UA. Estructuras textuales, artículos de investigación, caracterizaciones y ponencias. En: Sánchez UA. Manual de Redacción Académica e Investigativa: Cómo escribir, evaluar y publicar artículos. Medellín: Católica del Norte Fundación Universitaria; 2011. p. 147-50.

2. Campanario JM. Cómo escribir y publicar un artículo científico: cómo estudiar y argumentar su impacto. Rev Esp Doc Cient. 2003;26(4):461-3.

3. Day RA. Cómo escribir la introducción. En: Day RA. Cómo escribir y publicar trabajos científicos. M. Sáenz. (Trad.). 3era ed. Washington: Organización Panamericana de la Salud-OPS; 2005. p. 32-4.

4. Aranda TE. De la introducción. En: Aranda TE, Mitru TN, Costa AR. ABC de la redacción y publicación médico-científica. 2da ed. Madrid: Cooperación Cultural Exterior; 2009. p. 73-6.

5. Cohen C. Guía de redacción científica. CIRAD 2013. [Consultado el 10 de marzo del 2018] Disponible en: https://coop-ist.cirad.fr/actualites/guia-de-redaccion-cientifica

6. International Committee of Medical Journal Editors. Recommendations for the conduct, reporting, editing, and publication of scholarly work in medical journals (updated december 2017). [Consultado el 10 de marzo 2018]. Disponible en: http://www.icmje.org/recommendations/.
7. Villagran AT, Harris PR. Algunas claves para escribir correctamente un artículo científico. Rev Chil Pediatr. 2009;80(1):70-8.

8. Weinberger CJ, Evans JA, Allesina S. Ten Simple (Empirical) Rules for Writing Science. PLoS Comput Biol. 2015;11(4):1-6.https://doi.org/10.1371/journal. pcbi. 1004205

9. Moher D, Hopewell S, Schulz KF, Montori V, Gøtzsche PC, Devereaux PJ, et al. CONSORT 2010 explanation and elaboration: updated guidelines for reporting parallel group randomised trials. Int J Surg. 2012;10(1):2855. doi: 10.1016/j.ijsu.2011.10.001.

10. Simera I, Moher D, Hirst A, Hoey J, Schulz KF, Altman DG. Transparent and accurate reporting increases reliability, utility, and impact of your research: reporting guidelines and the EQUATOR Network. BMC Med. 2010;26(8):24. doi: 10.1186/1741-7015-8-24.

11. Moher D, Liberati A, Tetzlaff J, Altman DG, The PRISMA Group. Preferred Reporting Items for Systematic Reviews and Meta-Analyses. PLoS Med. 2009;6(7): e1000097. doi:10.1371/journal.pmed 1000097

12. Collado VS. Redacción científica: Algunos errores frecuentes. Biociencias. 2006;4:3-8.

13. Vílchez CM, Vara AA. Manual de redacción de artículos científicos. Instituto de Investigación de Ciencias Administrativas y Recursos Humanos. Universidad de San Martín de Porres. 2009.

14. CARE. Case report guidelines. [Consultado el 10 de abril del 2018]. Disponible en: http://www.care-statement.org/

15. Vandenbroucke JP, von Elm E, Altman DG, Gøtzsche PC, Mulrow C, Pocock SJ et al. Mejorar la comunicación de estudios observacionales en epidemiología (STROBE): explicación y elaboración. Gac Sanit. 2009;23(2):158e1-158e28. http://dx.doi.org/10.1590/ S0213-91112009000200015. 